\title{
Regulación del flujo sanguíneo uterino. I. Funciones de estrógeno y receptores estrogénicos $\alpha / \beta$ en el endotelio vascular uterino durante el embarazo
}

\author{
Mayra Pastore R., BSc. ${ }^{1,2}$, Rosalina Villalón L., BSc. ${ }^{1,2}$, Gladys López, MSc. ${ }^{2,3}$, \\ Jesús Iruretagoyena ${ }^{3,5}$, Ronald Magness, PhD. 1,2,3,4
}

${ }^{1}$ Programa de Endocrinología y Fisiología Reproductiva, ${ }^{2}$ Laboratorio de Investigación Perinatal, Departamento de Obstetricia y Ginecología y División de Ciencias de la Reproducción, ${ }^{3}$ Departamento Perinatal, ${ }^{4}$ Departamento de Ciencias de Animales, ${ }^{5}$ Departamento de Obstetricia y Ginecología de la División de Medicina Materno-Fetal.Universidad de Wisconsin-Madison, Wisconsin, USA.

Fuente de financiación: Este trabajo fue financiado por los Institutos Nacionales de Salud AA19446, HL49210, HD41921, HD38843, HL87144, R25GM083252 and 5T32HD041921. Parcialmente adaptado de Pastore MB et al. Semin Reprod Med 2012;30;46-61.

\section{RESUMEN}

El estrógeno y los receptores estrogénicos clásicos (REs), RE- $\alpha$ y RE- $\beta$, han demostrado ser parcialmente responsable por las adaptaciones endoteliales uterinas durante el embarazo al corto y largo plazo. Las diferencias moleculares y estructurales, junto con los diferentes efectos causados por estos receptores en las células y los tejidos, sugieren que su función varía dependiendo de la manera en la cual el estrógeno se comunica con sus receptores. En ésta revisión bibliográfica se discuten la función del estrógeno y sus receptores clásicos en las adaptaciones cardiovasculares durante el embarazo y la expresión de los Res in vivo e in vitro en el endotelio de la arteria uterina durante el ciclo ovárico y el embarazo, a la vez comparado con la expresión en endotelio arterial de tejidos reproductivos y no reproductivos. Estos temas integran el conocimiento actual de este amplio campo científico con interpretaciones e hipótesis diversas relacionadas con los efectos estrogénicos mediados bien sea por uno o los dos REs. Esta revisión también incluye la relación con las adaptaciones vasodilatadoras y angiogénicas requeridas para modular el dramático incremento fisiológico en la perfusión útero-placentaria observada durante un embarazo normal.

\section{PALABRAS CLAVE: Receptor de estrógeno, endotelio uterino, vasodilatación, angiogénesis, embarazo}

\section{SUMMARY}

Estrogen and classical estrogen receptors (ERs), ER- $\alpha$ and ER- $\beta$, have been shown to be partially responsible for short and long term uterine endothelial adaptations during pregnancy. The molecular and structural differences, together with the various effects caused by these receptors in cells and tissues, suggest that their function varies depending upon estrogen and estrogen receptor signaling. In this review, we discuss the role of estrogen and its classic receptors in the cardiovascular adaptations during pregnancy and the expression of ERs in vivo and in vitro in the uterine artery endothelium during the ovarian cycle and pregnancy, while comparing their expression in arterial endothelium from reproductive and non-reproductive 
tissues. These themes integrate current knowledge of this broad scientific field with various interpretations and hypothesis that related estrogenic effects by either one or both ERs. This review also includes the relationship with vasodilator and angiogenic adaptations required to modulate the dramatic physiological increase to the uteroplacental perfusion observed during normal pregnancy.

KEY WORDS: Estrogen receptor, uterine endothelium, vasodilatation, angiogenesis, pregnancy

\section{INTRODUCCIÓN}

El estrógeno produce sus efectos vasculares a través de sus receptores clásicos (REs), RE- $\alpha$ y $\mathrm{RE}-\beta$ en el endotelio vascular (1). A pesar de que estos receptores parecen ser similares, son estructural y funcionalmente distintos $(2,3)$. Las reacciones estrogénicas en el endotelio son clasificadas como genómicas (clásica/nuclear) y no-genómicas (no-clásica/membrana). En la vía clásica, el estrógeno se une a los receptores nucleares regulando la transcripción de genes $(4,5)$. Estos receptores nucleares son los más abundantes, mientras que los Res localizados en la membrana celular son menos abundantes (5\% de RE totales). Los receptores localizados en la membrana celular controlan la activación rápida de la enzima endotelial que media la producción del óxido nítrico (eNOS) y por lo tanto la vasodilatación causada por el óxido nítrico (ON) (5).

Estudios recientes han dilucidado la regulación compleja ejercida por los REs específicos en el lecho vascular uterino. Esto explica las adaptaciones cardiovasculares durante el embarazo. En esta revisión, centramos nuestra atención específicamente en eventos vasodilatadores rápidos mediados por ON, así como en reacciones angiogénicas uterinas de largo plazo mediadas por los RE- $\alpha$ y/o RE$\beta$. Las reacciones a largo plazo son colectivamente responsables de la manera espectacular en la que el flujo sanguíneo se eleva en la unidad útero-placentaria durante la gestación (6-8). Se evaluará la información relacionada con el papel que juega el estrógeno y los REs en las adaptaciones cardiovasculares durante el embarazo y la expresión de REs in vivo e in vitro en el endotelio de la arteria uterina durante el ciclo ovárico y el embarazo, contrastado con la expresión en el endotelio arterial de tejidos reproductivos y no-reproductivos.

El objetivo de esta revisión es integrar, a manera de resumen, las evidencias que apoyan el papel vital que ejercen los REs endoteliales en las adaptaciones vasculares uterinas inducidas por el embarazo, así como demostrar la heterogeneidad estructural, funcional y espacial de los REs.
El rol del estrógeno y sus receptores en las adaptaciones cardiovasculares durante el embarazo.

Durante la gestación normal, el flujo sanguíneo uterino (FSU) aumenta de manera sustancial, aproximadamente 50 veces más alto, con el fin de proporcionar suficientes nutrientes y oxígeno para el crecimiento y función saludable de la placenta y el feto (9). Esta adaptación vascular tan dramática tiene importancia biológica y clínica debido a que un incremento del flujo insuficiente del FSU produce una escasez de nutrientes y oxígeno lo cual podría estar asociado con la restricción del crecimiento intrauterino del feto y por ende un incremento en la morbilidad y mortalidad fetal y neonatal (10). El mecanismo a través del cual el estrógeno aumenta el FSU ha sido establecido en modelos tanto in vivo como in vitro. Estudios in vivo en ovejas gestantes y no gestantes demostró que la administración de estrógeno exógeno aumentó el FSU, confirmando que existe un aumento paralelo en los niveles circulantes de estrógeno y el FSU durante la fase folicular del ciclo ovárico y durante el embarazo normal (11-15). Existe también literatura que demuestra que los aumentos en el FSU inducidos por el estrógeno asociado con el embarazo son mediados en gran parte a través del aumento de expresión y actividad de eNOS (7,16-18). Estos cambios resultan en una regulación positiva en la producción endotelial del potente vasodilatador ON (8,19-22). No obstante, los mecanismos adicionales que describen los aumentos de FSU mediado por el estrógeno durante el embarazo también incluyen la estimulación de procesos diversos de angiogénesis. Tanto las reacciones vasodilatadoras como angiogénicas de la vasculatura uterina son influenciadas por el estrógeno a través de los REs por lo tanto el aumento en FSU inducido por el estrógeno pareciera ser mediado por uno o ambos REs $(23,24)$. En ovejas ooforectomizadas, se reportó que los aumentos en FSU inducidos por estrógeno exógeno fueron inhibidos en un $\sim 70 \%$ por el antagonista no-específico de los REs ICI 182,780, demostrando que los aumentos de FSU están par- 
cialmente influenciados por mecanismos dependientes de los REs (6). La administración unilateral de ICI 182,780 en arterias uterinas durante la fase folicular del ciclo ovárico y el embarazo, dos estados con altos niveles de estrógeno endógeno, redujeron locamente el FSU (6). Un estudio con evidencia preliminar demostró que ligandos selectivos del RE- $\alpha$ son más potentes que los ligandos de RE- $\beta$ para aumentar FSU. Este estudio fue conducido usando un modelo fisiológico ovino el cual apoya la premisa de que la vasodilatación mediada por estrógenos en el útero puede ser influenciada más por RE-a que por RE- $\beta$ (25). Por lo tanto, esta hipótesis requiere pruebas más rigurosas en el lecho vascular uterino.

Clásicamente, las funciones biológicas que ejercen los estrógenos en los tejidos, se cree que están mediadas por un RE especifico nuclear de alta afinidad y que funciona como un factor de transcripción para regular la expresión de genes (26). Sin embargo, la activación rápida de los mecanismos de señalización extracelular tales como las reguladas por señales quinasas (ERK) podrían ser un mecanismo adicional que es responsable por la activación rápida de eNOS a través del estrógeno para producir ON en las células endoteliales de las arterias uterinas (CEAU) in vitro. Anteriormente, hemos demostrado que la activación rápida de eNOS-ON mediada por el estrógeno en CEAUs ocurre a través de los REs localizados en la membrana plasmática (5).

Independientemente de la localización celular de los dos tipos de REs identificados hasta ahora el RE- $\alpha$ fue el primer receptor descrito mientras que RE- $\beta$ fue descubierto posteriormente (20,27-30). En este análisis se presenta información detallada acerca de la expresión de los RE- $\alpha$ y RE- $\beta$ en el endotelio de las arterias uterinas (AUs) y de la regulación que ejerce el estrógeno endógeno sobre el aumento en el FSU durante el embarazo. Este análisis se realizó midiendo los niveles de ARNm y proteínas de los RE- $\alpha$ y RE- $\beta$ en el endotelio de las arterias uterinas AUs (endoAU) in vivo y también en CEAUs obtenidas de ovejas y cultivadas in vitro.

\section{La expresión de los receptores estrogénicos in vivo e in vitro en el endotelio arterial uterino}

Nuestro grupo ha realizado varios análisis informáticos para estudiar los perfiles de expresión de los RE- $\alpha$ y RE- $\beta$ en AUs durante el ciclo ovárico y el embarazo ovino. Hemos sugerido que los niveles de expresión de los RE- $\alpha$ y/o RE- $\beta$ tienden a variar bajo diferentes condiciones fisiológicas y situaciones clínicas $(31,32)$. Es importante entender la configuración específica de los REs ya que esta es la clave en la regulación de su función biológica y por lo tanto en los efectos clínicos que produce. Varios grupos de investigación han documentado la presencia de los REs en arterias uterinas y otros tejidos reproductivos y no-reproductivos, incluyendo nuestro propio laboratorio $(5,20,30-38)$. En este estudio presentamos pruebas que apoyan la hipótesis de que los RE- $\alpha$ y RE- $\beta$ están presentes en endoAU de oveja y en el músculo liso vascular (MLV). Estos REs son regulados durante el ciclo ovárico/embarazo a través de alteraciones en los niveles de esteroides ovario/placentarios (estrógeno/progesterona).

Para determinar la localización celular de los RE- $\alpha$ y RE- $\beta$ en AUs (endo versus MLV), lo primero que se realizó fue la hibridación in situ del ARNm usando ${ }^{35}$ S-rótulo-en-sentido (control) o ribosoma anti-sentido sintetizado específicamente a cADNs del RE- $\alpha$ de oveja y el RE- $\beta$ de bovino (30) (Figura $1 \mathrm{~A}$ y $1 \mathrm{~B}$, respectivamente). La hibridación con sondas anti-sentido reveló que el ARNm del RE- $\alpha$ y $\mathrm{RE}-\beta$ están fuertemente presentes en el endotelio a lo largo de la túnica íntima de la AU ovina (es decir, en el lumen de los vasos). Como era de esperar, la expresión de los REs fue también observada en toda la túnica media. Los cortes de tejido sometidos a la hibridación con sonda en sentido mostraron niveles de fondo mínimo del ARNm del RE- $\alpha$ y de RE- $\beta$. También informamos que los cortes de tejido de la $\mathrm{AU}$ obtenidos durante los últimos meses de gestación (días 120 a 130; entre 0,8 a 0,9 del embarazo humano), mostraron señales inmunoreactivas intensas de las proteínas $R E-\alpha$ y $R E-\beta$ en el núcleo, y un nivel más bajo en el citoplasma de las células endoteliales y del músculo liso $(5,20)$ (Figura $2 \mathrm{~A}$ y $2 \mathrm{~B}$, respectivamente). Sin embargo, la detección de la señal inmunológica positiva correspondiente al RE- $\alpha$ y RE- $\beta$ en el citoplasma puede en parte derivados por la señal positiva de tinción inmunológica de la membrana plasmática debido a que esta técnica de inmunohistoquímica no proporciona una clara distinción de la estructura subcelular específicamente entre citoplasma versus la membrana plasmática. En estudios posteriores, se demostró que el RE- $\alpha$ se expresa de manera uniforme en las células de la AU (tinción inmunológica positiva dentro y fuera del núcleo), mientras que el RE- $\beta$ exhibe una señal de tinción inmunológica intensa preferencialmente en el núcleo. Estas observaciones son consistentes con otros experimentos realizados en células endoteliales pulmonares derivados de fetos bovino y en CEAUs de ovejas $(2,20,30,39)$. 


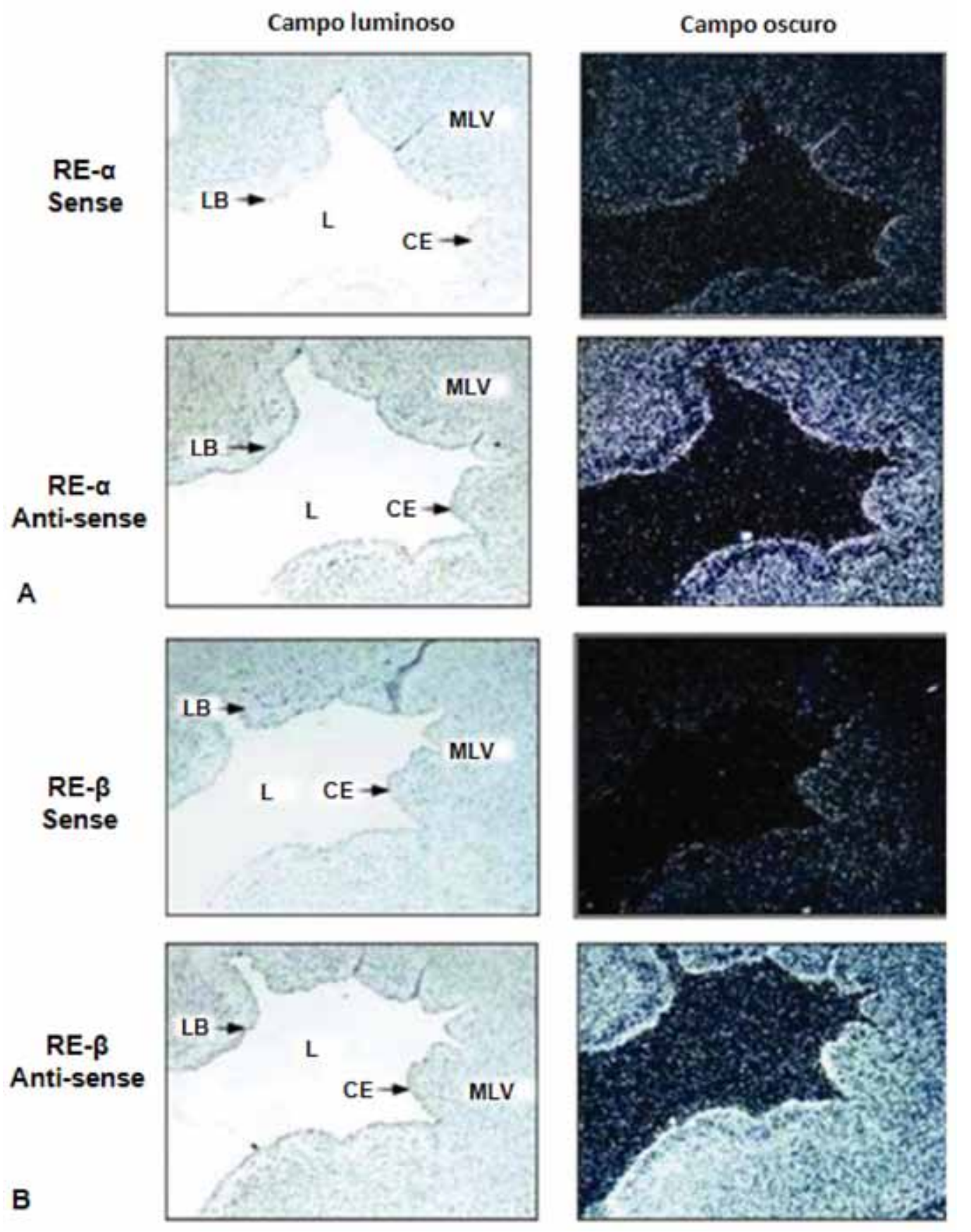

Figura 1. Ubicación del ARNm de los receptores de estrógeno (A) RE- $\alpha$ y (B) RE- $\beta$ en las arterias uterinas de ovina. Las arterias uterinas de ovejas gestantes fueron fijadas, embebidas en parafina y cortadas. La localización del ARNm de los RE- $\alpha$ y RE- $\beta$ se determinó mediante hibridación in situ utilizando ${ }^{35}$ S-rótulode-sentido (control) o ribosoma anti-sentido sintetizado específicamente a ADNcs del RE- $\alpha$ de ovina y el RE- $\beta$ de bovina. Las imágenes tomadas son representantes de cada grupo $(n)$ estudiado, $n=7$ de $10 x$ aumentos. $\mathrm{LB}=$ lámina basal, $\mathrm{CE}=$ células endoteliales, $\mathrm{L}=$ lumen, $\mathrm{MLV}=$ músculo liso vascular (imágenes reproducidas con permiso de Byers MJ, et al. J Physiol 2005;565:85-99). 

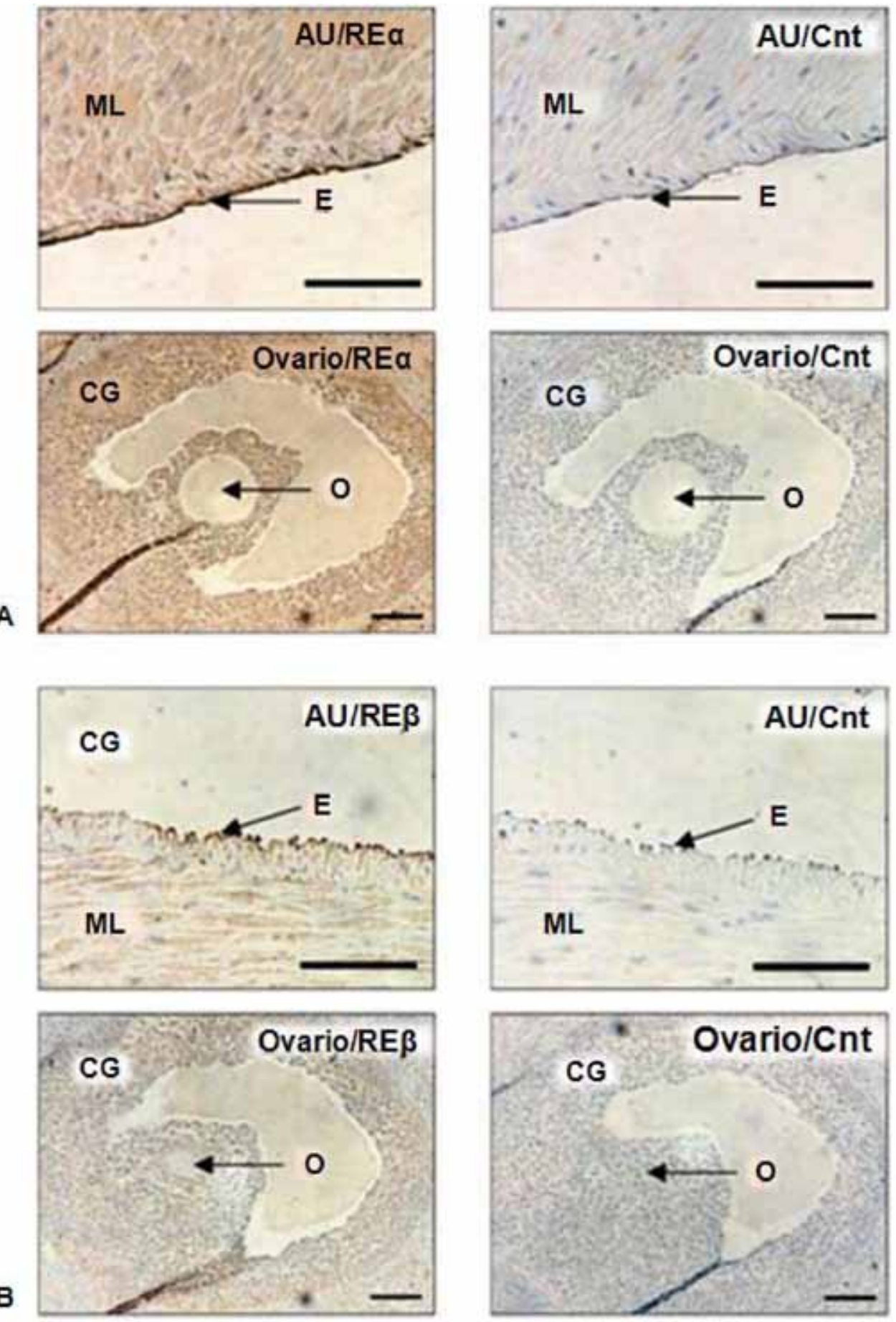

Figura 2. El análisis inmunohistoquímico del (A) RE- $\alpha$ y (B) RE- $\beta$ en la arteria uterina de las ovejas gestantes. Tejido ovárico de las mismas ovejas se utilizó como un control positivo para RE- $\alpha$ y RE- $\beta$ que muestra una expresión de proteína abundante tanto de RE- $\alpha$ y RE- $\beta$ en las células granulosas, pero no en el resto del ovario. $E$ = endotelio; $C G=$ células de la granulosa, $O=$ oocitos, $M L=$ músculo liso. Barra $=$ de 100 micras (imágenes reproducidas con permiso de Liao WX et al. Biol Reprod 2005;72:530-7). 
Utilizando el análisis de inmunotransferencia Western, que es diferente a las técnicas histológicas descritas anteriormente por ser más cuantitativo, se realizaron estudios para determinar si la expresión de las proteínas de los RE- $\alpha$ y/o RE- $\beta$ se modificaban en los endotelios reproductivos a diferencia de los no-reproductivos durante estados fisiológicos de niveles altos de estrógeno (fase folicular y el embarazo) y progesterona (fase lútea y el embarazo) (Figura 3). Los cambios de niveles de proteína de los RE- $\alpha$ y RE- $\beta$ en el endoAU se compararon con endotelio obtenido de otros tejidos reproductivos, como la mama (endoMA) y la placenta (endoPL) así como endotelio de tejidos no-reproductivos, incluyendo el omento (endoOA), riñón (endoRA) y arteria coronaria (endoCO). El resultado de estos experimentos demostró un incremento del $50 \%$ en la expresión del RE- $\alpha$ en el endoAU durante la fase folicular versus el endoAU en la fase lútea. No hubo diferencias significativas en la expresión del RE- $\alpha$ en ninguna otra preparación endotelial durante la fase folicular (Figura $3 A)$, lo cual demuestra no sólo la especificidad de estos cambios al nivel del RE- $\alpha$ en el endoAU, sino el control local que los ovarios tienen sobre la vasculatura uterina. Por la excepción de endoAU y posiblemente (discreta tendencia) en el endoMA, el RE- $\beta$ no demostró cambios significativos en ninguna de las preparaciones foliculares endoteliales de los tejidos no-reproductivos que fueron estudiados en comparación con la fase lútea (Figura 3B). Por el contrario, durante el embarazo, los tres endotelios aislados de tejidos reproductivos mostraron un aumento significativo en el nivel de expresión del RE- $\beta$. En concreto, el RE- $\beta$ del endoAU del grupo de embarazo se incrementó un $60 \%$ mientras que el endoMA se incrementó un $50 \%$ sobre los valores del grupo control en la fase lútea. Además, los vasos uterinos y mamarios mostraron ser muy sensibles a la vasodilatación mediada por el estrógeno $(9,40)$. Es probable que a pesar de la falta de cambio de RE- $\beta$ durante la fase folicular, el incremento de la expresión de RE- $\beta$ durante la gestación es debido a la prolongada exposición de estos endotelios al estrógeno y progesterona derivados de la placenta.

El endotelio placentario demostró tener un $230 \%$ mayor expresión de RE- $\beta$ que el endoAU obtenido en la fase lútea y sustancialmente mayor expresión en el endoAU obtenido durante el embarazo. Debido a que la expresión del RE- $\alpha$ en endoPL fue menor que en endoAU en la fase lútea, pareciera indicar que el RE- $\beta$ es el RE principal en la musculatura placentaria ovina. Colectivamente, estas observaciones apuntan a la posibilidad de que el RE- $\beta$ posee un papel único en la regulación de las funciones vasculares durante el desarrollo del embarazo. El único tejido no-reproductivo en el cual la expresión de RE- $\beta$ se incrementó de manera significativa, un $50 \%$ por encima del valor de la fase lútea, fue el endoCO. En el endoMA y endo$\mathrm{CO}$, los niveles calculados durante la fase folicular sugieren una tendencia de estos lechos vasculares de ser sensibles al estrógeno. Varios grupos científicos han demostrado múltiples comparaciones de expresión de los REs en tejidos vasculares (41-50). En nuestros estudios se informó sobre proteínas aisladas de varios endotelios que han sido ampliamente estudiadas con respecto al ciclo endocrino del animal.

Aunque el RE- $\alpha$ fue fácilmente detectado en los vasos sanguíneos analizados, ninguno de los otros endotelios de tejido (reproductivos o no-reproductivos) estudiados, excepto los del endoAU y endo$P L$, demostraron niveles de RE- $\alpha$ significativamente diferentes durante la fase lútea (Figura 3A). Los niveles de RE- $\beta$ tampoco fueron alterados por el ciclo ovárico/embarazo en los lechos vasculares de endoRA y endoOA; lechos tradicionalmente considerados como los centros principales que controlan la presión arterial. La observación de que los dos REs no fueron alterados en endoOA (datos no mostrados), por el ciclo ovario, la ooforectomía con o sin terapia de reemplazo hormonal, puede tener una implicación importante ya que hemos demostrado que el estrógeno aumenta profundamente el FSU en un 100-200\% tan rápido como en 2 horas, mientras que el flujo de sangre omental permanece inalterado $(6,7,23)$. Hay que destacar que el RE- $\beta$ (Figura $3 B$ ) se elevó en los tres endotelios de tejidos reproductivo ( $\mathrm{AU}, \mathrm{MA}, \mathrm{y} \mathrm{PL})$ durante el embarazo así como en el endoCO. Adicionalmente, los niveles del RE- $\beta$ durante la fase folicular fueron similares a los niveles observados durante el embarazo en tejidos de $\mathrm{AU}, \mathrm{MA}$ y $\mathrm{CO}$. Así que el endoCO fue el único endotelio derivado de tejidos no-reproductivos que mostró la capacidad de regular el nivel del RE- $\beta$, lo cual sugiere un papel más ubicuo para este receptor en la vasculatura mamífera tal como lo sugieren estudios con ratones RE- $\beta$ knockout (KO). Los ratones RE- $\beta$ KO desarrollaron enfermedades cardiovasculares hipertensivas vinculadas con la edad (51). El hallazgo de que el RE- $\beta$ en endoCO aumenta durante el embarazo es particularmente interesante, dado el aumento sustancial del gasto cardíaco, el ritmo cardíaco, el volumen sistólico y la profunda disminución de la resistencia vascular sistémica observada durante el embarazo $(7,23,52)$. Además, la cantidad de sangre que irriga los lechos vasculares evaluados puede variar con 


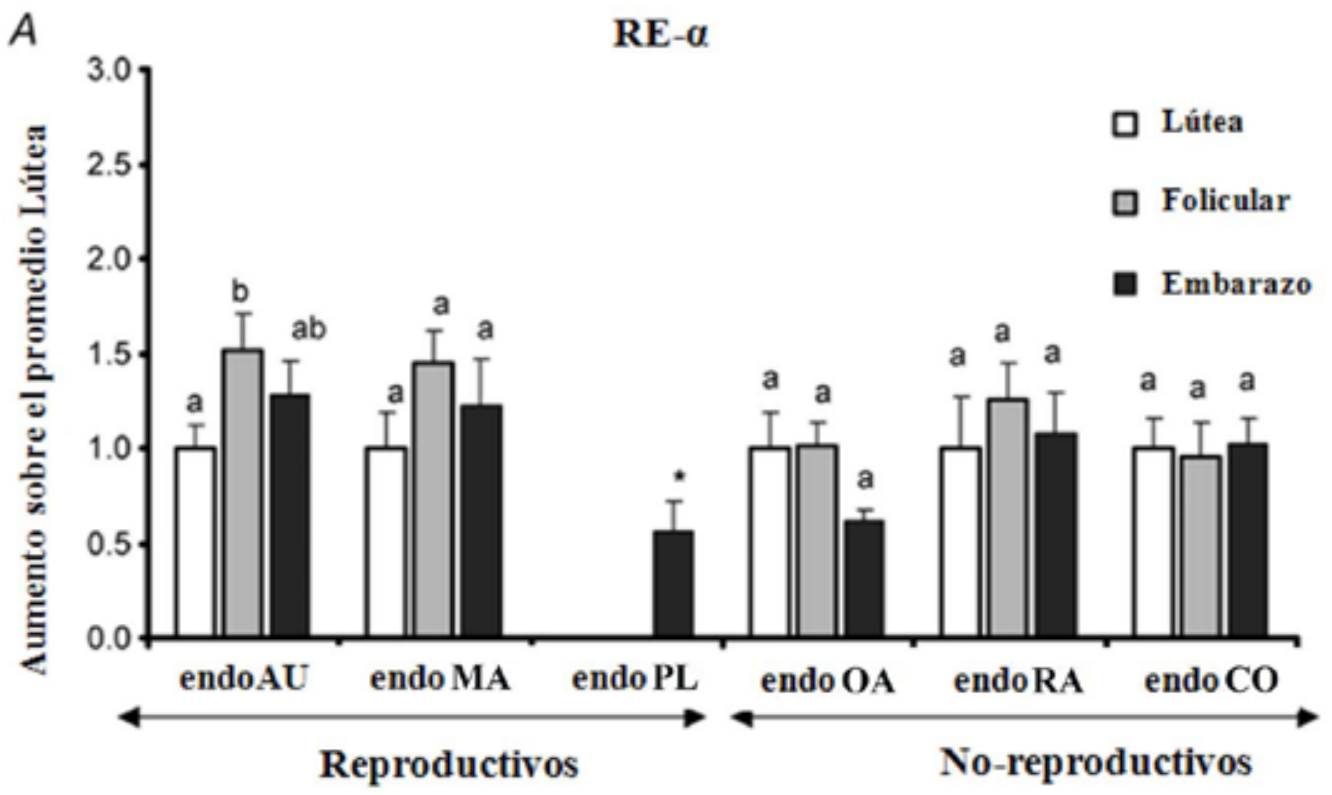

$B$

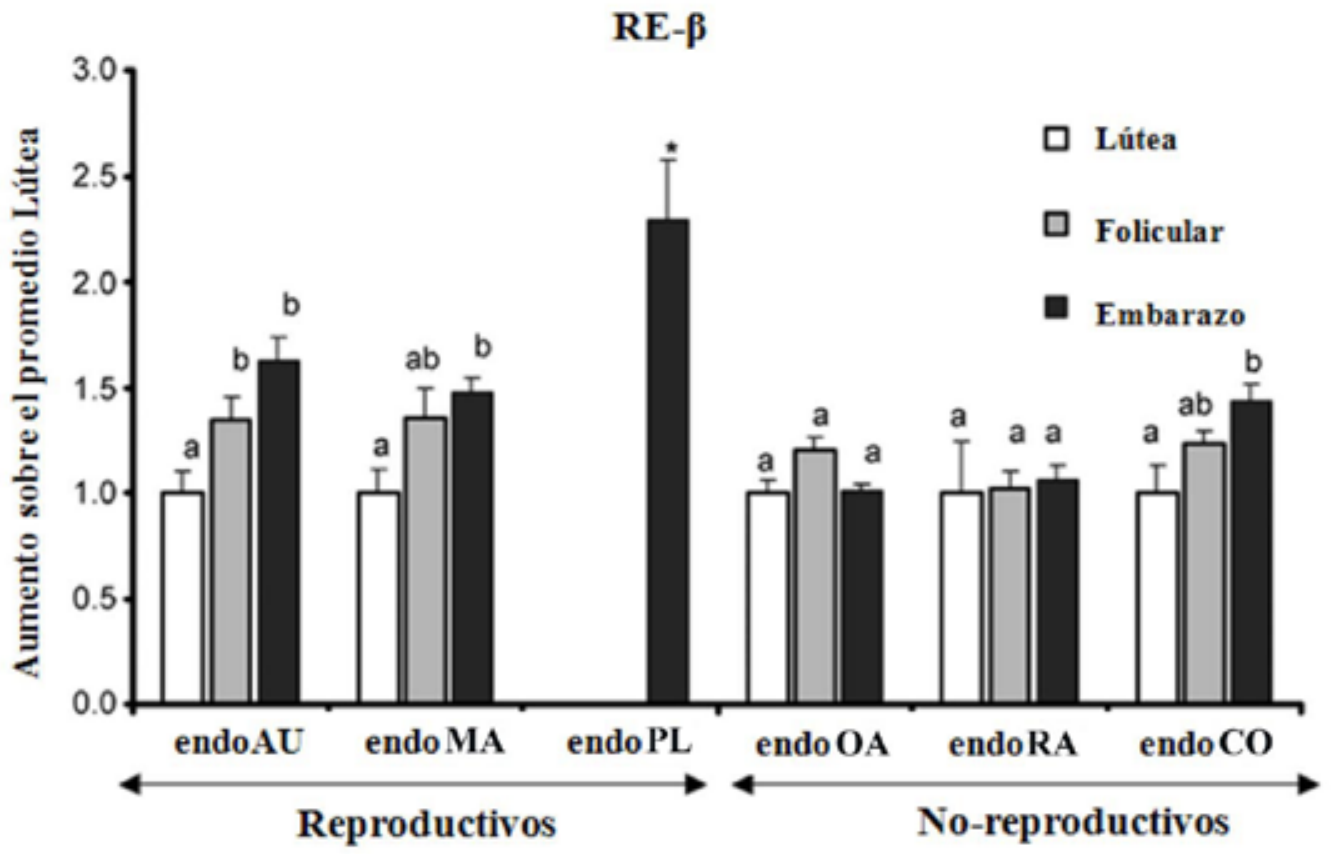

Figura 3. Evaluación de niveles de expresión relativos del (A) RE- $\alpha$ y (B) RE- $\beta$ en endotelios arteriales de tejidos reproductivos y no-reproductivos utilizando análisis de Western blot. Las diferencias en expresión en los especímenes en fase lútea, folicular, y gestante en oveja se ilustran como un aumento (fold) sobre el promedio de todas las muestras en fase lútea de un tipo de arteria, analizadas en el mismo Western blot. El endoPL muestra doble expresión comparado con el promedio del endoAU en fase lútea. Los datos representan el promedio +/- el error estándar del mismo. Los promedios con las letras diferentes son estadísticamente significativos $(\mathrm{p}<0,05)$ (imágenes reproducidas con permiso de Byers MJ, et al. J Physiol 2005;565:85-99). 
el tratamiento exógeno con $\mathrm{E}_{2} \beta$. El flujo sanguíneo se incrementó en los lechos vasculares uterinos, mamarios, y coronarios, pero no en los lechos del omento o renales $(7,18,40)$. Por otra parte, el aumento en el FSU, coronario y mamario inducido por el estrógeno son atenuados por el tratamiento con el inhibidor de NOS, L-NAME (8,13,53-55). En este sentido, eNOS fue positivamente regulado en el endoAU durante la folículogénesis y el embarazo, aunque está presente en todos los endotelios reproductivos y no-reproductivos $(19,30,56,57)$. Estos datos prueban que la activación de eNOS y por lo tanto la producción de ON, es más importante en el endotelio CO y MA que la elevación en la capacidad de eNOS. De igual manera se refuerza la naturaleza única del endo $\mathrm{AU}$ como un tejido muy sensible al estrógeno en comparación con otros lechos vasculares.

Para validar y establecer un modelo in vitro de las acciones estrogénicas sobre el endotelio vascular, hemos buscado demostrar la presencia de los REs en CEAUs cultivadas. En primer lugar, medimos el nivel de expresión de proteína de RE- $\alpha$ en las membranas plasmáticas y extractos nucleares aislados de CEAUs usando Western Blot y realizamos estudios usando $E_{2} \beta-B S A-F I T C$ (agonista del estrógeno que es impermeable a la membrana celular y es marcado con fluorescencia) (5). En nuestros experimentos observamos la presencia de la proteína clásica del RE- $\alpha$ de 67-kDa en la membrana plasmática y el extracto nuclear. Sin embargo, como se esperaba, el mayor nivel relativo de la proteína fue encontrado en el núcleo en comparación con la membrana plasmática. Por otra parte, los estudios de acoplamiento usando $E_{2} \beta-B S A-F I T C$ mostraron que la señal fue localizada principalmente en la membrana plasmática de CEAUs. La localización de ambos RE- $\alpha$ y RE- $\beta$ en CEAUs fue confirmada usando inmunocitoquímica (Byers y Magness, observaciones no publicadas). La expresión de los RE- $\alpha$ y RE- $\beta$ también fueron confirmadas por los análisis de Western (Figura 4) y reacción de polimerasa en tiempo real (no mostrado) en CEAUs. Estos datos nos llevan a la conclusión de que estas preparaciones de CEAUs de cultivo primario proporcionan un modelo útil in vitro para los estudios de las acciones del estrógeno en la función vascular uterina y el mecanismo de señalización.

Debido a que el RE- $\beta$ aparenta ser el subtipo de receptor principal que mostró cambios en la $A U$ $y$ en otros endotelios reproductivos durante la gestación, también se evaluaron las variantes del RE- $\beta$ en CEAUs. La expresión de diversos variantes del
RE- $\beta$ han sido identificados en tejidos reproductivos humanos, pero actualmente solo hay datos limitados que puedan explicar sus funciones, por lo cual no existen datos disponibles en el estudio del endotelio de la arteria uterina. Sin embargo, la variante truncada del RE- $\beta 2$ ha sido detectada en el endoAU (EAU) y en CEAUs. Esta variante se ha postulado como un regulador negativo de las acciones estrogénicas. La presencia de este variante demuestra que el RE- $\beta 2$ no sólo se encuentra presente ex vivo, sino que también se ha mantenido elevada en CEAUs (Figura 4).

Desde un punto de vista estructural, el RE- $\beta 2$ es el resultado de la supresión del empalme en el exón 5 , le faltan todos los aminoácidos en el terminal-C y carecen del ligand binding domain (LBD) que es parte de RE- $\beta(58,59)$. Otros estudios han demostrado que puede servir como un receptor negativo capaz de bloquear tanto la señalización de RE- $\alpha$ como de RE- $\beta$, lo que le convierte en una herramienta a través de la cual los niveles de expresión de los dos receptores podrían ser manipulados. En nuestros estudios no evaluamos los niveles de la variante RE- $\beta 2$ en el endoAU durante la fase lútea o folicular, aunque la hipótesis sigue siendo que durante la fase lútea los niveles de la variante RE- $\beta 2$ serían más altos que durante la fase folicular o del embarazo. Si este es el caso, es posible que este RE dominante negativo pudiera ayudar a explicar la incrementación de RE- $\alpha$ y/o RE- $\beta$ durante la fase folicular y el embarazo (Figura 3). Independientemente, su papel en las acciones del estrógeno y el embarazo sigue siendo desconocido y requiere un examen más detallado.

\section{CONCLUSIÓN}

Este resumen presenta datos que apoyan la idea de que el estrógeno y los REs están involucrados en las adaptaciones endoteliales a corto y largo plazo durante el embarazo. El aumento del FSU es controlado en parte por los niveles cíclicos de estrógeno endógeno. Estudios in vivo han demostrado que las proteínas de los RE- $\alpha$ y RE- $\beta$ son expresadas en los endotelios de las arterias de tejidos reproductivos y no-reproductivos y su patrón de expresión cambia en endotelios reproductivos dependiendo de los niveles de estrógeno presente. Estudios ex vivo demostraron la presencia de sólo el RE- $\beta$ en el endotelio del endometrio, en todas las fases del ciclo ovárico en primates humanos y no humanos, lo que apunta a efectos estrogénicos que son genómicos y no genómicos orquestado por 
el RE- $\beta$ en la ausencia del RE- $\alpha$. Por otra parte, la localización de los RE- $\alpha$ y RE- $\beta$ en la membrana citoplasmática o en el núcleo celular también indican funciones distintas. Estos resultados, sugieren que la investigación de la señalización estrogénica

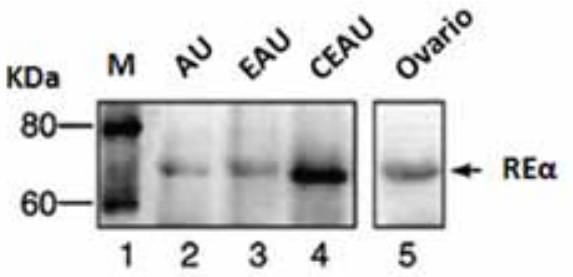

A

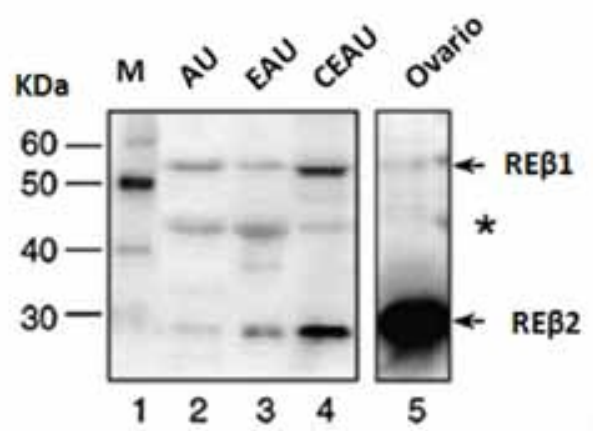

B

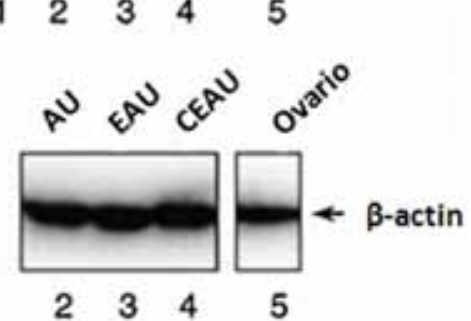

C

$\begin{array}{llll}2 & 3 & 4 & 5\end{array}$

\section{Epitope de anticuerpo}

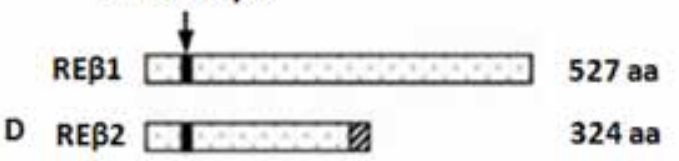

Figura 4. Análisis de Western inmunoblot del (A) RE- $\alpha$, (B) RE- $\beta$, y (C) $\beta$-actina en los extractos de proteínas de la arteria uterina (AU), endotelio arterial uterino (EAU), células endoteliales de la arteria uterina (CEAU) y ovario de ovejas gestante. (D) Diagrama que representa la forma truncada del RE- $\beta 2$ que resulta por la eliminación del splicing del exón 5 (panel B). El cuadro sombreado representa las secuencias de aminoácidos codificados por un marco de lectura diferente. aa=aminoácido. Las bandas marcadas con asterisco pueden indicar formas truncadas adicionales de RE- $\beta$ (imágenes reproducidas con permiso de Liao WX, et al. Biol Reprod 2005;72:530-7). requiere un conocimiento específico tanto de la heterogeneidad funcional de cada subtipo de receptor, así como de la localización de estos. Estas diferencias podrían explicar los efectos divergentes del estrógeno en las células y tejidos. En conclusión, todos los aspectos que se discutieron en este resumen sobre el papel de los receptores estrogénicos en la adaptación al embarazo, podrían ayudar a entender los signos clínicos de patología vascular que son evidentes en las etapas finales del embarazo.

Reconocimiento: Queremos dar las gracias a Cindy L. Goss por la ayuda en la preparación de esta revisión.

\section{REFERENCIAS}

1. Meyers, M.J., Sun, J., Carlson, K.E., Marriner, G.A., Katzenellenbogen, B.S., Katzenellenbogen, J.A. Estrogen receptor-beta potency-selective ligands: structure-activity relationship studies of diarylpropionitriles and their acetylene and polar analogues. J Med Chem 2001;44;4230-51.

2. Mendelsohn, M.E., Karas, R.H. The protective effects of estrogen on the cardiovascular system. $\mathrm{N}$ Engl $\mathrm{J}$ Med 1999;340;1801-11.

3. Pastore, M.B., Jobe, S.O., Ramadoss, J., Magness, R.R. Estrogen receptor-alpha and estrogen receptorbeta in the uterine vascular endothelium during pregnancy: functional implications for regulating uterine blood flow. Semin Reprod Med 2012;30:4661.

4. Chambliss, K.L., Yuhanna, I.S., Mineo, C., Liu, P., German, Z., Sherman, T.S., et al. Estrogen receptor alpha and endothelial nitric oxide synthase are organized into a functional signaling module in caveolae. Circ Res 2000;87:E44-52.

5. Chen, D.B., Bird, I.M., Zheng, J., Magness, R.R. Membrane estrogen receptor-dependent extracellular signal-regulated kinase pathway mediates acute activation of endothelial nitric oxide synthase by estrogen in uterine artery endothelial cells. Endocrinology 2004;145:113-25.

6. Magness, R.R., Phernetton, T.M., Gibson, T.C., Chen, D.B. Uterine blood flow responses to $\mathrm{ICl} 182780$ in ovariectomized oestradiol-17beta-treated, intact follicular and pregnant sheep. J Physiol 2005;565;7183.

7. Magness, R.R., Phernetton, T.M., Zheng, J. Systemic and uterine blood flow distribution during prolonged infusion of 17beta-estradiol. Am J Physiol 1998;275:H731-43.

8. Rosenfeld, C.R., Cox, B.E., Roy, T., Magness, R.R. Nitric oxide contributes to estrogen-induced vasodilation of the ovine uterine circulation. J Clin Invest 1996;98:158-66.

9. Rosenfeld, C.R. Distribution of cardiac output in ovine pregnancy. Am J Physiol 1977;232:H231-5.

10. Magness, R.R., Rosenfeld, C.R. The role of steroid hormones in the control of uterine blood flow. In The 
Uterine Circulation, C.R. Rosenfeld, ed. (Ithaca, NY: Perinatology Press), 1989; pp. 239-71.

11. Ford, S.P. Control of uterine and ovarian blood flow throughout the estrous cycle and pregnancy of ewes, sows and cows. J Anim Sci 1982;55 Suppl 2:32-42.

12. Ford, S.P., Christenson, R.K. Blood flow to uteri of sows during the estrous cycle and early pregnancy: local effect of the conceptus on the uterine blood supply. Biol Reprod 1979;21:617-24.

13. Gibson, T.C., Phernetton, T.M., Wiltbank, M.C., Magness, R.R. Development and use of an ovarian synchronization model to study the effects of endogenous estrogen and nitric oxide on uterine blood flow during ovarian cycles in sheep. Biol Reprod 2004;70:1886-94.

14. Greiss, F.C., Jr., Anderson, S.G. Uterine vescular changes during the ovarian cycle. Am J Obstet Gynecol 1969;103:629-40.

15. Harvey, C.A., Owen, D.A. Changes in uterine and ovarian blood flow during the oestrous cycle in rats. $J$ Endocrinol 1976;71:367-9.

16. Greiss, F.C., Jr., Anderson, S.G. Effect of ovarian hormones on the uterine vascular bed. Am J Obstet Gynecol 1970;107:829-36.

17. Rosenfeld, C.R., Morriss, F.H., Jr., Makowski, E.L., Meschia, G., Battaglia, F.C. Circulatory changes in the reproductive tissues of ewes during pregnancy. Gynecol Invest 1974;5:252-68.

18. Rosenfeld, C.R., Killam, A.P., Battaglia, F.C., Makowski, E.L., Meschia, G. Effect of estradiol-17, on the magnitude and distribution of uterine blood flow in nonpregnant, oophorectomized ewes. Pediatr Res 1973;7:139-48.

19. Magness, R.R., Rosenfeld, C.R., Hassan, A., Shaul, P.W. Endothelial vasodilator production by uterine and systemic arteries. I. Effects of ANG II on PGI2 and NO in pregnancy. Am J Physiol 1996;270:H1914-23.

20. Liao, W.X., Magness, R.R., Chen, D.B. Expression of estrogen receptors-alpha and -beta in the pregnant ovine uterine artery endothelial cells in vivo and in vitro. Biol Reprod 2005;72:530-7.

21. Yi, F.X., Boeldt, D.S., Gifford, S.M., Sullivan, J.A., Grummer, M.A., Magness, R.R., et al. Pregnancy enhances sustained $\mathrm{Ca} 2+$ bursts and endothelial nitric oxide synthase activation in ovine uterine artery endothelial cells through increased connexin 43 function. Biol Reprod 2010;82:66-75.

22. Salhab, W.A., Shaul, P.W., Cox, B.E., Rosenfeld, C.R. Regulation of types I and III NOS in ovine uterine arteries by daily and acute estrogen exposure. Am J Physiol Heart Circ Physiol 2000;278:H2134-42.

23. Magness, R.R., Rosenfeld, C.R. Local and systemic estradiol-17 beta: effects on uterine and systemic vasodilation. Am J Physiol 1989;256:E536-42.

24. Rosenfeld, C.R., Roy, T., Cox, B.E. Mechanisms modulating estrogen-induced uterine vasodilation. Vascul Pharmacol 2002;38:115-25.

25. Baker RS, H.J., Friedman A, Clark KE. ER alpha receptors mediate estrogen induced increases in uterine blood flow. J Soc Gynecol Investig 2003;10:332.
26. Mangelsdorf, D.J., Thummel, C., Beato, M., Herrlich, P., Schutz, G., Umesono, K., et al. The nuclear receptor superfamily: the second decade. Cell 1995;83:835-9.

27. Green, S., Walter, P., Kumar, V., Krust, A., Bornert, J.M., Argos, P., et al. Human oestrogen receptor cDNA: sequence, expression and homology to v-erb-A. Nature 1986;320:134-9.

28. Greene, G.L., Gilna, P., Waterfield, M., Baker, A., Hort, Y., Shine, J. Sequence and expression of human estrogen receptor complementary DNA. Science 1986;231:1150-4.

29. Kuiper, G.G., Enmark, E., Pelto-Huikko, M., Nilsson, S., Gustafsson, J.A. Cloning of a novel receptor expressed in rat prostate and ovary. Proc Natl Acad Sci USA 1996;93:5925-30.

30. Byers, M.J., Zangl, A., Phernetton, T.M., Lopez, G., Chen, D.B., Magness, R.R. Endothelial vasodilator production by ovine uterine and systemic arteries: ovarian steroid and pregnancy control of ERalpha and ERbeta levels. J Physiol 2005;565:85-99.

31. Fujimoto, J., Hirose, R., Sakaguchi, H., Tamaya, T. Clinical significance of expression of estrogen receptor alpha and beta mRNAs in ovarian cancers. Oncology 2000;58:334-41.

32. Lindner, V., Kim, S.K., Karas, R.H., Kuiper, G.G., Gustafsson, J.A., Mendelsohn, M.E. Increased expression of estrogen receptor-beta mRNA in male blood vessels after vascular injury. Circ Res 1998;83:224-9.

33. Giambiagi, N., Pasqualini, J.R., Greene, G., Jensen, E.V. Recognition of two forms of the estrogen receptor in the guinea-pig uterus at different stages of development by a monoclonal antibody to the human estrogen receptor. Dynamics of the translocation of these two forms to the nucleus. J Steroid Biochem 1984;20:397-400.

34. Batra, S., losif, S. Nuclear estrogen receptors in human uterine arteries. Gynecol Obstet Invest 1987;24:250-5.

35. Wu, W.X., Ma, X.H., Smith, G.C., Nathanielsz, P.W. Differential distribution of ERalpha and ERbeta mRNA in intrauterine tissues of the pregnant rhesus monkey. Am J Physiol Cell Physiol 2000;278:C190-8.

36. Weihua, Z., Saji, S., Makinen, S., Cheng, G., Jensen, E.V., Warner, M., et al. Estrogen receptor (ER) beta, a modulator of ERalpha in the uterus. Proc Natl Acad Sci USA 2000;97:5936-41.

37. Chang, K., Lubo, Z. Steroid hormones and uterine vascular adaptation to pregnancy. Reprod Sci 2008;15:336-48.

38. Malamas, M.S., Manas, E.S., McDevitt, R.E., Gunawan, I., Xu, Z.B., Collini, M.D., et al. Design and synthesis of aryl diphenolic azoles as potent and selective estrogen receptor-beta ligands. J Med Chem 2004;47:5021-40.

39. Chambliss, K.L., Shaul, P.W. Rapid activation of endothelial NO synthase by estrogen: evidence for a steroid receptor fast-action complex (SRFC) in caveolae. Steroids 2002;67:413-9.

40. Rosenfeld, C.R., Morriss, F.H., Jr., Battaglia, F.C., 
Makowski, E.L., Meschia, G. Effect of estradiol-17beta on blood flow to reproductive and nonreproductive tissues in pregnant ewes. Am J Obstet Gynecol 1976;124:618-29.

41. Kuiper, G.G., Carlsson, B., Grandien, K., Enmark, E., Haggblad, J., Nilsson, S., et al. Comparison of the ligand binding specificity and transcript tissue distribution of estrogen receptors alpha and beta. Endocrinology 1997;138:863-70.

42. Taylor, A.H., Al-Azzawi, F. Immunolocalisation of oestrogen receptor beta in human tissues. $\mathrm{J} \mathrm{Mol}$ Endocrinol 2000;24:145-55.

43. Karas, R.H., Patterson, B.L., Mendelsohn, M.E. Human vascular smooth muscle cells contain functional estrogen receptor. Circulation 1994;89:1943-50.

44. Losordo, D.W., Kearney, M., Kim, E.A., Jekanowski, J., Isner, J.M. Variable expression of the estrogen receptor in normal and atherosclerotic coronary arteries of premenopausal women. Circulation 1994;89:1501-10.

45. Venkov, C.D., Rankin, A.B., Vaughan, D.E. Identification of authentic estrogen receptor in cultured endothelial cells. A potential mechanism for steroid hormone regulation of endothelial function. Circulation 1996;94:727-33.

46. Kim-Schulze, S., McGowan, K.A., Hubchak, S.C., Cid, M.C., Martin, M.B., Kleinman, H.K., et al. Expression of an estrogen receptor by human coronary artery and umbilical vein endothelial cells. Circulation 1996;94:1402-7.

47. Caulin-Glaser, T., Watson, C.A., Pardi, R., Bender, J.R. Effects of 17beta-estradiol on cytokine-induced endothelial cell adhesion molecule expression. J Clin Invest 1996;98:36-42.

48. Hodges, Y.K., Tung, L., Yan, X.D., Graham, J.D., Horwitz, K.B., Horwitz, L.D. Estrogen receptors alpha and beta: prevalence of estrogen receptor beta mRNA in human vascular smooth muscle and transcriptional effects. Circulation 2000; 101:1792-8.

49. Andersson, C., Lydrup, M.L., Ferno, M., Idvall, I., Gustafsson, J., Nilsson, B.O. Immunocytochemical demonstration of oestrogen receptor beta in blood vessels of the female rat. J Endocrinol 2001;169:241-7.
50. Orimo, A., Inoue, S., Ikegami, A., Hosoi, T., Akishita, M., Ouchi, Y., et al. Vascular smooth muscle cells as target for estrogen. Biochem Biophys Res Commun 1993;195:730-6.

51. Zhu, Y., Bian, Z., Lu, P., Karas, R.H., Bao, L., Cox, D., et al. Abnormal vascular function and hypertension in mice deficient in estrogen receptor beta. Science 2002;295:505-8.

52. Libby, P., Bonow, R.O., Mann, D.L., Zipes, D.P. Pregnancy and Cardiovascular Disease. In Braunwald's Heart Disease: A Textbook of Cardiovascular Medicine, Volume Single. (Philadelphia: Saunders Elsevier), 2008; p. 1967.

53. Buren, G.A.V., Yang, D.-s., Clark, K.E. Estrogeninduced uterine vasodilatation is antagonized by L-nitroarginine methyl ester, an inhibitor of nitric oxide synthesis. Am J Obstet Gynecol 1992;3:828-33.

54. Lang, U., Baker, R.S., Braems, G.A., Clark, K.E. Inhibition of nitric oxide (NO) synthesis antagonises the oestrogen-induced increase in coronary blood. Eur J Cancer 2000;36 Suppl 4:111.

55. Mershon, J.L., Baker, R.S., Clark, K.E. Estrogen increases iNOS expression in the ovine coronary artery. Am J Physiol Heart Circ Physiol 2002;283:H1169-80.

56. Magness, R.R., Sullivan, J.A., Li, Y., Phernetton, T.M., Bird, I.M. Endothelial vasodilator production by uterine and systemic arteries. VI. Ovarian and pregnancy effects on eNOS and $\mathrm{NO}(\mathrm{x})$. Am J Physiol Heart Circ Physiol 2001;280:H1692-8.

57. Vagnoni, K.E., Shaw, C.E., Phernetton, T.M., Meglin, B.M., Bird, I.M., Magness, R.R. Endothelial vasodilator production by uterine and systemic arteries. III. Ovarian and estrogen effects on NO synthase. Am J Physiol 1998;275:H1845-56.

58. Hirata, S., Shoda, T., Kato, J., Hoshi, K. Isoform/ variant mRNAs for sex steroid hormone receptors in humans. Trends in endocrinology and metabolism: TEM 2003;14:124-9.

59. Inoue, S., Hoshino, S., Miyoshi, H., Akishita, M., Hosoi, T., Orimo, H., et al. Identification of a novel isoform of estrogen receptor, a potential inhibitor of estrogen action, in vascular smooth muscle cells. Biochem Biophys Res Commun 1996;219:766-72. 